\title{
CEFALÉIA CERVICOGÊNICA
}

\author{
RELATO DE UM CASO
}

MAURICE VINCENT * - GUNNAR BOVIM **

\begin{abstract}
RESUMO - A produção de cefaléias a partir de anormalidades no pescoço nunca foi sistematizada a ponto de permitir o estudo de sua patogenia, embora fosse fato conhecido há muitos anos. Sjaastad et al. descreveram, em 1983, grupo de pacientes com uma cefaléia uniforme e estereotipadla. Os ataques se caracterizavam por dor moderada, unilateral sem mudança de lado, de longa duração, recorrendo em poucas semanas. A cefaléia podia ser provocada por certos movimentos do pescoço ou por compressão de pontos desencadeadores ali localizados. A dor se iniciava rua região cervical, irradiando-se para as áreas órbito-fron-ᄀ to-temporal e/ou facial. A denominação «cefaléia cervicogênica» (CC) foi proposta para esta entidade. Sua fisiopatogenia não é completamente conhecida. Bloqueios da raiz C2 e do nervo grande occipital eliminam a dor. Apresentamos um caso de CC, fazendo alguns comentários acerca do seu quadro clínico, fisiopatologia e tratamento.
\end{abstract}

\section{Cervicogenic headache: report of a case}

SUMMARY - It has been known for many years that headaches can originate from abnormalities in the neck. However, their clinical pictures were never sufficiently systematized, at least not in order to permit the research on their pathogenesis. Sjaastad et al. described in 1983 a group of patients with a very uniform and stereotyped headache. Attacks of mild, longlasting, unilateral head pain without sideshift, occurred every few weeks. The headache could be provoked by neck movements, such as extension, rotation or lateral flexion, as well as by external pressure towards trigger points in the neck. It usually started back in the neck, eventually spreading to the ipsilateral orbito-frontal-temporal or facial areas. The denomination «cervicogenic headache» ( $\mathrm{CH}$ ) was proposed. Its pathophysiology is presently unknown. The $\mathrm{C} 2$ and occipital nerve blockages eliminate the pain. We present a $\mathrm{CH}$ case and make some comments on its clinical picture, pathophysiology, and treatment.

O conhecimento de que algumas cefaléias podem ser desencadeadas por anormalidades no pescoço não é recente. Sua descrição, entretanto, nunca foi sistematizada a ponto de permitir o diagnóstico diferencial com outras cefaléias, bem como o estudo de sua etiologia e patogenia 17. Segundo Bártschi-Rochaix, Schützenberger (1853) foi o primeiro a descrever uma cefaléia causada por afecções nas vértebras cervicais i. Barre publicou, em 1926, estudo acerca do que chamou de «syndrome sympathique cervicale postérieur», composta por cefaléia, distúrbios vestibulares e visuais, passando a ser conhecida como síndrome de Barré-Lieou. Vários relatos surgiram desde então, cada qual invocando a participação de dada estrutura cervical, como vértebras, discos intervertebrais. articulações uncovertebrais, artérias vertebrais e raízes cervicais 5. A doença é provavelmente idêntica à que foi identificada por Hunter e Mayfield, em 1949 8. Sjaastad et al. relataram, em 1983, grupo de pacientes com uma síndrome uniforme, caracterizada por cefaléia estereotipada associada a

Trabalho realizado no Hospital da Universidade de Trondheim, Noruega: * Neurologista, Hospital Universitário Clementino Fraga Filho, Universidade Federal do Rio de Janeiro (Serviço do Prof. Sérgio Novis); ** Neurologista, Hospital da Universidade de Trondheim (Regionsykehuset i Trondheim), Noruega (Serviço do Prof. Ottar Sjaastad).

Dr. Maurice Vincent - Rua General Glicério 183/301 - 22251 Rio de Janeiro RJ - Brasil. 
mecanismos desencadeadores no pescoço, tendo sido proposta a denominação de cefaléia cervicogênica $(\mathrm{CC}) ?,{ }^{25}$. Sua real existência e denominação ainda são controvertidas. A CC não faz parte da última classificação internacional das cefaléias 10,22.

Apresentamos um caso de CC, fazendo alguns comentários acerca de seu quadro clínico, fisiopatologia e tratamento.

\section{OBSERVAÇÃO}

JAB, paciente do sexo feminino, branca, natural da Noruega, com 34 anos de idade. Sua história se iniciou em setembro-1967, quando foi vítima de acidente automobilístico. Seu pescoço sofreu intensa flexão lateral para o lado esquerdo (E), após a qual notou rigidez à movimentação da coluna cervical. Foi recomendado o uso de colar cervical por 4 semanas. Sete dias após o tnauma passou a apresentar episódios de cefaléia de 3 a 4 dias de duração, não-pulsátil, de intensidade moderada, mais pronunciada na região retro-ocular e no pescoço, exclusivamente do lado $E$. Os ataques apresentaram, com o tempo, clara tendência a croniíaacfto. No primeiro ano havia longos períodos de remissão, da ordem de semanas. A partir de setembro-1989 a cefaléia tornou-se praticamente contínua, de intensidade variada, eventualmente interrompida por poucos dias sem dor. Quando a dor era mais intensa, associa vam-se fenômenos autonômicos do tipo náuseas, raramente vômitos, injeção conjuntival, além de foto e fonofobia moderadas. Os ataques eram acompanhados de desconforto no membro superior E. Não havia relação entre o aparecimento da dor e o consumo de bebidas alcoólicas, tonteiras, dificuldade para engolir ou desconforto na garganta. A extensão e a rotação da coluna cervical para ambos os lados, por exemplo ao estacionar automóvel, podiam provocar o aparecimento da cefaléia unilateral ou, mesmo, desencadear ataque de vãrios dias de duração. A história patológica pregressa foi negativa; sempre que apresentava cefaléia antes do acidente, mesmo que muito raramente, a dor era global e responsiva a analgésicos comuns; negava outras doenças na família, incluindo enxaqueca ou outras formas de cefaléia. Ao exame, a mobilidade do pescoço estava reduzida, principalmente no que se refere à extensão e rotação para a $E$; a palpação do pescoço à $E$ mostrou-se dolorida em vários pontos, principalmente n»a suposta localização do nervo occipital maior (dois centímetros lateralmente à protuberância occipital externa e 2-4 centímetros inferiormente) e da raiz C2 (posteriormente ao processo mastóide); os demais itens do exame físico geral e neurológico foram normais. Radiografias panorâmicas da coluna cervical, ressonância nuclear magnética do crânio e coluna cervical e flebografia da órbita E foram normais. Não houve resposta à administração intravenosa de ergotamina ou inalação de oxigênio a $100 \%$, 7Lí/min, por máscara. Como prova diagnostica, seu nervo occipital maior $\mathbf{E}$ foi infiltrado com $2 \mathrm{ml}$ de xilocaina a $1 \%$ a cefaléia e o desconforto no braço desapareceram completamente e a mobilidade do pescoço aumentou. Foi realizado novo bloqueio com xilocaina e betametazona que resultou no desaparecimento dos sintomas por 3 meses. A raiz C2 foi também bloqueada, resultando alívio completo da dor.

\section{COMENTÁRIOS}

A dor na CC é unilateral, sem alternância de lados. Os ataques são relativamente raros, mas pode haver com o tempo certa tendência a cronificação. A dor permanece tipicamente 1 a 3 dias, podendo durar de horas a mais de 1 semana. O intervalo sem dor dura 1 a 4 semanas, podendo ser de 2 dias a 2 meses. Seu caráter é não-pulsátil, eventualmente latejante, de intensidade moderada, exigindo por vezes repouso no leito. A dor se inicia na região occipital, irradiando-se para a região órbito-fronto-temporal ou facial (Fig. I)'?. De fato, este é um dado importante na diferenciação entre CC e migrânia (enxaqueca). Não há predominância noturna. Fases mais amenas e mais graves podem durar anos. O envolvimento do pescoço é caracterizado por, pelo menos, um dos seguintes critérios: localização da dor também na região da nuca, ombro ou braço; história de trauma cervical prévio; possibilidade de alguns pacientes de terem suas crises desencadeadas por movimentação do pescoço (Tabela 1). Estacionar um carro ou limpar o teto são procedimentos tipicamente desencadeadores. Frequentemente um acidente de automóvel, levando a movimentação brusca da coluna cervical, é o trauma que dá origem à doença. O sexo feminino é mais afetado na proporção de 2 para 1 . A doença pode iniciar-se entre os 6 e 40 anos ou, mais frequentemente, em torno dos $30^{7}>^{11} »^{2} 5$.

SINAIS E SINTOMAS ASSOCIADOS - Mais de $50 \%$ dos pacientes têm mal-estar, náuseas e vômitos. É possível apresentarem desconforto na garganta, frequentemente descrito como «secura»7. Visão turva, sem escotomas e ipsilateral, é referida por 

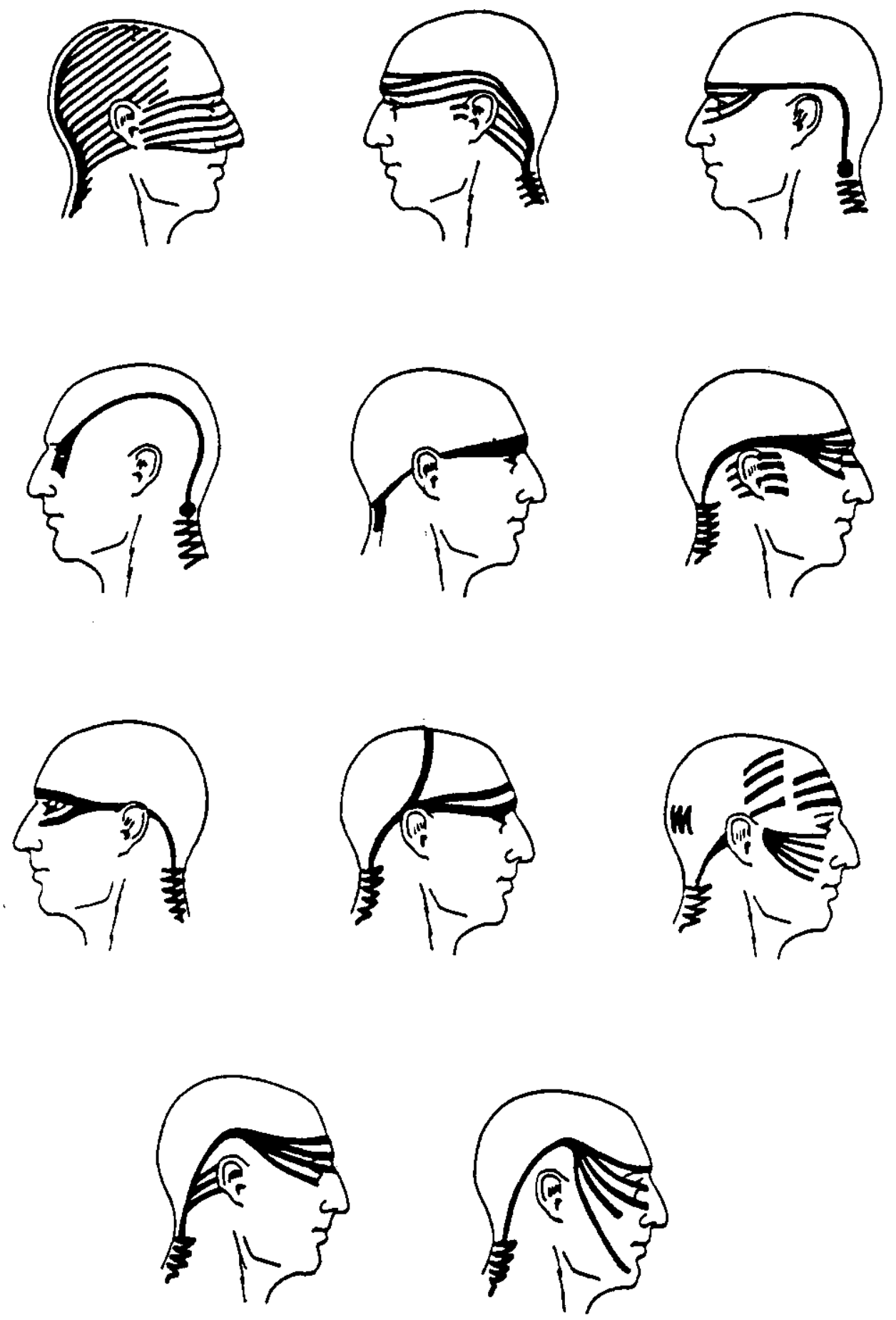

Frig. 1 - Irnadiacăo da dor em 11 pacientes com cefaleia cervicogenica. Modificado, com permissão, a partir de Ziredriksen et al. (Cervicogenic headache: clinical manifestation. Cephalalgia 1987, $7: 147$ $160)$.

Compressāo

Raiz C2

Processo transverso de C4, C5

Inserçōes musculares na protuberancia occipital externa

Ponto equidistante à protuberância occipital externa e mastóide

Nervo grande occipital

Movimentos da cabeca

Rotação

Flexão

Extensão

Inclinação lateral

Outros

Espirro

Tosse

Manobra de Valsalva, evacuação

Tabela 1 - Cefaléia cervicogênica; precipitacâa mecanica. 
alguns pacientes. Alterações autonômicas ipsilaterais, semelhantes às que ocorrem na cefaiéia em salvas (CS) («cluster headache») ou na hemicrania paroxística crônica (CPH), podem surgir acompanhando um ataque (Tabela 2)7,17,25. Sintomas premonitórios como sensação de latejamento no pescoço no lado da dor, «jabs-andjolts» 19 occipitais, visão turva ou desconforto na região da dor podem anteceder os ataques. O exame físico não raro demonstra rigidez e diminuição de amplitude à movimentação passiva do pescoço. Em um caso foi relatada hipoestesia nos territórios de inervação correspondente a C2 e C3 7.

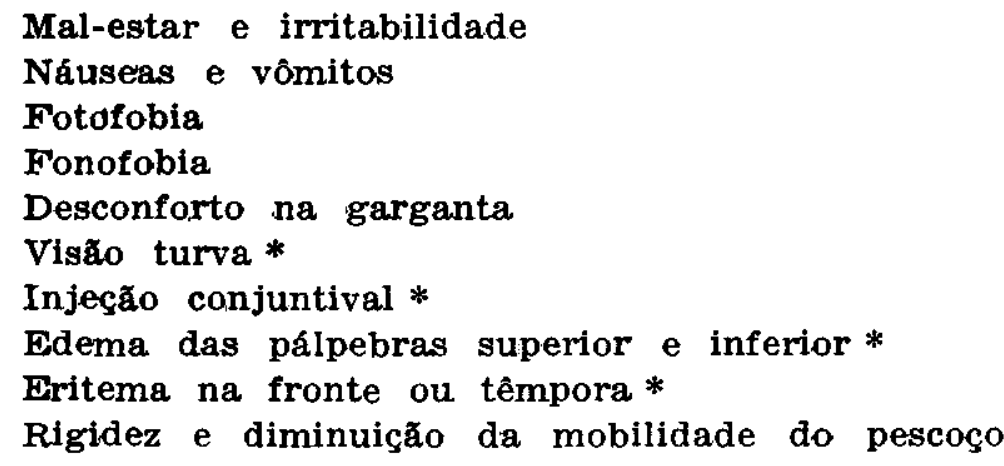

Tabela \& - Cefaléia cervicogenniaa: sintomas e sinais associados.

* Lado sintomatico.

INVESTIGAÇÃO COMPLEMENTAR - A sudorese, medida na fronte por evaporímetro durante ataques espontâneos, mostra simetria entre os lados sintomático e não-sintomático. Quanto à indução por calor, a sudorese é maior na CC que na CS, mas a supersensibilidade à pilocarpina é maior na segunda 4. A pupilometria mostra anisocorias que não diferem dos controles. A supersensibilidade à instilação de colírio de fenilefrina no lado sintomático, encontrada na $\mathrm{CS}$, não existe na $\mathrm{CC}^{8}$. Exames radiológicos (radiografias da coluna cervical, mielografias, tomografias computadorizadas e angiografias) são na maioria normais, ou apresentam alterações sem especificidade para a doença em questão 6.

DIAGNÓSTICO DIFERENCIAL - A diferenciação entre CC e migrânia é particularmente difícil. Os quatro principais fatores decisivos a serem considerados estão na Tabela $3 \mathbf{7 , 2 2 , 2 3 , 2 5}$. $\hat{\mathbf{E}}$ provável que muitos pacientes tidos como enxaquecosos com má resposta terapêutica na verdade sejam casos de CC. Em segundo lugar, o diagnóstico diferencial deve ser feito com outras cefaléias unilaterais, como CS, CPH, hemicrania contínua (HC) e a recém-descrita «shortlasting unilateral neuralgiform headache attacks with conjunctival injections and tearing», para a qual propomos a denominação «S U NCT» (Tabela*).

\begin{tabular}{lll}
\hline Dor & Cefaléia cervicogênica & Migrânia \\
$\begin{array}{l}\text { Unilateral, } \\
\text { sem mudança de lado. }\end{array}$ & $\begin{array}{l}\text { Unilateral, } \\
\text { com mudança de lado }\end{array}$ \\
$\begin{array}{l}\text { Possivel irradiação } \\
\text { Alterações visuais }\end{array}$ & Unilaterais para a fronte & Bilaterais \\
$\begin{array}{l}\text { Critérios de } \\
\text { envolvimento do pescoço }\end{array}$ & Presentes & Ausentes a nuca
\end{tabular}

Tabela $3-$ Cefaléia cervicogenica $\times$ migrania. 


\begin{tabular}{|c|c|c|c|c|c|c|}
\hline & $\mathrm{CC}$ & $\mathrm{Cs}$ & CPH & $\mathbf{H C}$ & SUNCT & EC \\
\hline Sexo & fem $>$ masc & masc $>$ fem & fem $>$ masc & fem $>$ masc & masc(?) & fem $>$ masc \\
\hline Unilateralidade & $+t+$ & ++ & $++t$ & ++ & $+t$ & - \\
\hline Duração & dias/sem & $\min /$ horas & $\min$ & dias/contínua & seg & horas/dias \\
\hline Frequêencia & + & ++ & $+t+$ & continua & $+t+t$ & + \\
\hline Intensidade & $+(+)$ & $+t+$ & $+t+$ & $+(+)$ & $+t$ & $+(+)$ \\
\hline $\begin{array}{c}\text { Precipitação } \\
\text { mecânica }\end{array}$ & + & - & + & - & $?$ & 一 \\
\hline PS & - & ++ & + & - & + & $一$ \\
\hline DAF & $+(-)$ & $+t+$ & $+t+$ & + & $+t+t$ & - \\
\hline $\begin{array}{l}\text { Localização } \\
\text { (máxima dor) }\end{array}$ & $\begin{array}{l}\text { Envolve } \\
\text { pescoço }\end{array}$ & Retro-ocular & $\begin{array}{l}\text { Oculo- } \\
\text {-frontal }\end{array}$ & Temporal & Olho & $\begin{array}{l}\text { Fronto- } \\
\text {-temporal }\end{array}$ \\
\hline
\end{tabular}

Tabela 4 - Diagnostico diferencial.

$C C$, cefaleia cervicogenica; CS, cefaleía em salvas; CPH, hemicrania paroxistica crónica; HC,

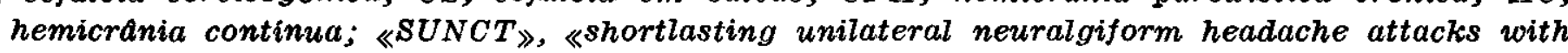
conjunctival injection and tearing》; $E C$, enxaqueca comum; $P S$, padrão em salvas; $D A F$, distúrbios autonómicos na face.

A CS é fácil de ser diferenciada pela preferência masculina, intensidade, duração e padrão de ocorrência dos paroxismos 12.15. Ataques menos severos de CS podem todavia ocorrer, permitindo que os pacientes continuem a exercer suas atividades normalmente, não obstante a presença da dor 27. $\mathrm{Na} \mathrm{CPH}$, os ataques são curtos, a intensidade da dor é muito grande, tal como na CS, e, principalmente, os ataques desaparecem completamente com o uso de indometacina 20,29. A HC inicialmente pode cursar com ataques intermitentes e, assim, confundir-se com CC. Porém, a resposta à indometacina é também absoluta neste caso. A localização da dor, mais na região ocular, pode ser um critério a favor de HC. Frequentemente, os pacientes referem dor no olho, e não atrás do olho, como na CS e na CPH 28.

Em 1977/78 Sjaastad et al. examinaram paciente que apresentava, havia muitos anos, ataques de dor na região súpero-medial da órbita direita, ao longo da ponte do nariz, de curta duração (10 a $20 \mathrm{seg}$, maiores do que neuralgia do trigêmio e menores que CPH) e frequência elevada (ataques a cada 2-3 minutos, centenas por dia). Havia intensa injeção conjuntival e lacrimejamento, bem como edema palpebral. Dois outros pacientes, também do sexo masculino, foram vistos posteriormente, sendo esta síndrome relatada em 1989 26. Como na neuralgia do trigêmeo, dois dos pacientes apresentavam zonas de gatilho na face ou tinham ataques ao se alimentar. Os aspectos clínicos são suficientes para o discrime entre «SUNCT» e CC.

Outras afecções podem ser lembradas, estas de diferenciação mais fácil. A «neck-tongue syndrome» deve ser suspeitada diante de pacientes com dor no pescoço e parestesias na hemilíngua, secundárias a movimentos bruscos da coluna cervical 13.17. A síndrome de Raeder cursa com dor na região frontal e Horner parcial, podendo haver comprometimento de nervos cranianos ao exame 9.17. Paroxismos de dor unilateral no ouvido irradiando-se para a mastóide, pescoço, nariz, palato e maxila, diminuição do paladar nos \% anteriores da língua e hipersecreção das glândulas lacrimal, submandibular e sublingual configuram a neuralgia do gânglio geniculado 17. A síndrome de Eagle é secundária a existência de longos processos estilóides; a dor é persistente, localizada na faringe e região lingual posterior 3,16. Em análise de 100 casos de carotidínia, Lovshin encontrou predominância no sexo feminino (4:1); a dor parte da região carotidiana e irradia-se para cima, no curso da carótida externa 1`»17. A arterite temporal acomete pacientes mais velhos e a velocidade de hemossedimentação está usualmente elevada; a dor é temporal e unilateral^. 
FISIOPATOGENIA - Não existem ainda evidências suficientes para permitir a determinação da etiologia da CC. O mérito desta denominação está exatamente no fato de considerar que ela é produzida no pescoço, sem que qualquer estrutura ou tecido seja responsabilizado em especial. Três razões suportam a idéia de que a CC nasce no pescoço: (a) os ataques podem ser provocados por movimentos ou compressão de pontos específicos; (b) a dor no braço, em alguns pacientes, pode ser radiculopática; (c) há, por vezes, rigidez e hipomotilidade ao exame ${ }^{25}$. A etiologia pode ainda ser multifatorial, envolvendo o nervo grande occipital, as raízes C1, C2 e C3, o plexo venoso circunvizinho, a artéria vertebral e seu plexo venoso 5. Jaeger sugere que a CC pode surgir de pontos desencadeantes específicos de dor miofascial, provocados por disfunções na coluna cervical, mas o mecanismo pelo qual produziria fenômenos autonômicos e dor referida não é explicado". Carella diz, em 1972, que anomalias no atlas podem provocar cefaléia unilateral, posterior com irradiação para o vértex e sensação de estiramento («stretching») atrás do olho ipsilateral, por irritação das primeiras raízes sensitivas cervicais ${ }^{2}$. Segundo Pfaffenrath, a raiz C2 carreia impulsos dolorosos provenientes de ligamentos, discos intervertebrais e nervos espinhais em fibras não-mielinizadas do tipo $C$; devido à relação anatômica estreita entre as 4 primeiras raízes cervicais e o trato do trigêmeo, a dor pode ser referida em áreas anteriores, principalmente VI mas, também, V2; arcos-reflexo conectando o trato espinhal com a glândula lacrimal, mucosa nasal e vasos da conjuntiva explicariam os fenômenos autonômicos faciais $\mathbf{1 8 .}$

TRATAMENTO - Os bloqueios percutâneos e as cirurgias têm sido propostos para cefaléias de origem cervical. As raízes C2 e C3 e o nervo grande occipital são as principais estruturas bloqueadas, com lidocaína, como prova diagnostica, e buvicaína ${ }^{2 *}{ }^{2} \wedge$. A uncoforaminectomia foi realizada com êxito por Gronbaek, em uma série (impura) de 26 pacientes. O método foi depois alterado por Jung et al. e Pasztor, com acesso ântero-lateral e $87 \%$ de sucesso 25. Segundo Sjaastad, Blume et al. têm empregado eletrocoagulação por radiofreqüência no território do nervo occipital, com bons resultados ${ }^{24}$. Um programa de tratamento não invasivo para dores miofasciais foi igualmente relatado como eficazli.

COMENTÁRIOS FINAIS - O capítulo de cefaléias desencadeadas no pescoço não tem merecido muita atenção nas publicações especializadas5, apesar de ser o pescoço importante na produção ou no desencadeamento desta e de outras cefaléias, como a CPH 20,21,29 A pesquisa acerca dos reais mecanismos que conectam estruturas cervicais e cranianas na produção de dor tornará possível o esclarecimento da fisiopatologia deste grupo de doenças e a abertura de novas perspectivas terapêuticas.

Agradecimento - Os autores agradecem os valiosos comentários do Prof. Ottar Sjaastad.

\section{REF E R Ê N CI A S}

1. Bartschi-Rochaix W. Headaches of cervical origin. In Vinken PJ, Bruyn GW (eds) : Handbook of Clinical Neurology. Amsterdam: North-Holland, 1968, Vol 5, p 192-203.

2. Carella A. Slight anomalies of the atlas: their pathogenetic meaning. Neuroradiology 1972, 3:224-227.

3. Eagle WW. Elongated styloid process: further observations and a new syndrome. Arch Otolaryngol 1948, 47:630-640.

4. Fredriksen TA. Cervicogenic headache: the forehead sweating pattern. Cephalalgia 1988, 8:203-209.

5. Fredriksen TA. Studies on Cervicogenic Headache. University of Trondheim, Department of Medicine. Trondheim: T A PIR, 1989.

6. Fredriksen TA, Fougner R, Tangerud A, Sjaastad O. Cervocogenic headache: radiological investigations concerning head/neck. Cephalalgia 1989, 9:1-8.

7. Fredriksen TA, Hovdahl H, Sjaastad O. ^Cervicogenic Headache»: clinical manifestation. Cephalalgia 1987, $7: 147-160$.

8. Fredriksen TA, Wysocka-Bakowska MM, Bogucki A, Antonaci F. Cervicogenic headache: pupillometric findings. Cephalalgia 1988, 8:93-103.

9. Grimson BS, Thompson HS. Raeder's syndrome: a clinical review. Surv Ophthalmol 1980, $24: 199-210$.

10. Headache Classification Committee of the International Headache Society. Classification and Diagnostic Criteria for Headache Disorders, Cranial Neuralgias and Facial Pain Cephalalgia $1988, \quad 8:($ S 7 ). 
11. Jaeger B. Are «cervicogenic» headaches due to myofascial plain and cervical spine dysfunction? Cephalalgia 1989, 9:157-164.

12. Krabbe A A. Cluster headache: a review. Acta Neurol Scand 1986, 74:1-9.

13. Lance JW, Antony M. Neck-tongue syndrome on sudden turning of the head. J Neurol Neurosurg Psychiat 1980, 43:97-101.

14. Lovshin LL. Carotidynia. Headache 1987, 17:192-195.

15. Mianzoni GC, Terzano MG, Bono G, Micieli G, Martucci N, Nappi G. Cluster headache: clinical findings in 180 patients. Cephalalgia 1983, 3:21-30.

16. Massey EW, J. Elongated styliod process (Eagle's syndrome) causing hemicrania. Headache 1979, $19: 339-344$.

17. Pfaffenrath V. Cervicogenic headache and its differential diagnosis. In Ferrari MD, Lataste X (eds): Migraine and Other Headaches. Park Ridge, USA. Parthenon, 1989, p $161-177$.

18. Pfaffenrath V, Dandekar R, Pöllmann W. Cervicogenic headache: the clinical picture, radiological findings and hypotheses on its pathophysiology. Headache 1987, 27:495-499.

19. Sjaastad O. Chronic paroxismal Hemicrania (CPH). In: Vinken PJ, Bruyn GW, Klawans HLi, Rose FC (eds): Handbook of Clinical Neurology. Amsterdam: Elsevier 1986, Vol 4 (48), p 257-266.

20. Sjaastad O, Dale I. Evidence for a new(?) treatable headache entity: a preliminary report. Headache 1974, $14: 105-108$.

21. Sjaastad O, Dale I. A new(?) clinical headache entity: chronic paroxysmal hemicrania. Acta Neurol Scand 1976, $54:$ 140-159.

22. Sjaastad O, Fredriksen TA, Sand T. The localization of the initial pain of attack: a comparison between classic migraine and cervicogenic headache. Functional Neurol 1989, $4: 1-6$.

23. Sjaastad O, Fredriksen TA, Sand T, Antonaci F. Unilaterality of headache in classic migraine. Cephalalgia 1989, 9:1-7.

24. Sjaastad O, Fredriksen TA, Stolt-Nielsen A. Cervicogenic headache, C2 rhizopathy, and occipital neuralgia: a connection? Cephalalgia 1986, 6:189-195.

25. Sjaastad O, Saunte C, Hovdahl H, Breivik H, GrOnbaek E. «Cervicogenic» headache: an hypothesis. Cephalalgia 1983, 3:249-256.

26. Sjaastad O, Saunte C, Salvesen R, Fredriksen TA, Seim A, Roe, OD, Fostad K, Lobben OP, Zhao MJ. Shortlasting, unilateral neuralgiform headache attacks with conjunctival injection, tearing, sweating and rhinorrhea. Cephalalgia 1989, 9:147-156.

27. Sjaastad O. de Souza Carvalho D, Zhao JM. «Mild» cluster Headache(?). Chephalalgia 1988, 8:121-126.

28. Sjaastad O, Spierings ELH. Hemicrania continua: another headache absolutely responsive to indomethacin. Cephalalgia 1984, 4:65-70.

29. Vincent M. Hemicrania paroxística crônica: aspectos clínicos. Rev Bras Neurol 1989 , $25: 69-75$. 